\title{
Optimization of ultrasonic-assisted extraction of inulin from Jerusalem artichoke
}

\author{
Lina Yang, Hongyun Zhang, Jinghang Huang, Yafan Zhao, Danshi Zhu, He Liu* \\ College of Food Science and Technology, Bohai University. Jinzhou, 121013, China
}

\begin{abstract}
An efficient ultrasonic-assisted extraction of inulin from Jerusalem artichoke was investigated by Response Surface Methodology (RSM). The results showed that the yield of ultrasonic-assisted extraction was $62.07 \pm 0.39 \%$ over the microwave $(40.85 \pm 0.28 \%)$ and hot water extraction $(27.42 \pm$ $0.42 \%$ ). The Box-Behnken Design (BBD) was used to optimize the effects of three parameters (temperature-X1, ultrasonic power-X2 and time-X3) on inulin yield. Analysis of variance showed that the contributions of X1, X3, X12, X13, X22 were significant. The optimal yield of inulin was $82.93 \pm 1.03 \%$ at $82{ }^{\circ} \mathrm{C}, 120 \mathrm{~W}$ and $18 \mathrm{~min}$.
\end{abstract}

\section{Introduction}

Inulin, a non-digestible carbohydrate, is natural functional dietary fibers for improving bowel health [1]. A significant work had been reported on determination of degree of polymerization and prebiotic effect evaluation of inulin from Jerusalem artichoke [2].

At present, hot water extraction mostly was used for the industrial production of inulin, but common problems were low extraction rate, excessive loss and quality instability [3]. Ultrasound-assisted extraction has a fast and efficient advantages and recognizes as an alternative approach to traditional extraction methods due to high-density, high-frequency sound waves and the role of promoting the dissolution of active ingredients [4]. Compared with conventional extraction, it not only accelerated the extraction rate, saved the extraction time and reduced the organic solvent waste, but also enhanced the efficiency and the quality [5].

Therefore, the significant variables (temperature, ultrasonic power and time) were investigated by Response Surface Methodology (RSM). By optimizing the inulin extraction, it could provide theoretical parameters for practical production.

\section{Materials and Methods}

\subsection{Materials}

Jerusalem artichoke was purchased from Jinzhou farm produce market, then crushed to pass through 40 mesh screen and stored at $-4{ }^{\circ} \mathrm{C}$ until used for further analysis. Other chemicals were all of analytical grade.

\subsection{Extraction of inulin}

The Jerusalem artichoke powder $(5.00 \mathrm{~g})$ was put into a $500 \mathrm{~mL}$ beaker and added the water (the liquid ratio of 1:20). The inulin was extracted in the ultrasonic cell disintegrator (SJIA-1200W, Ningbo Shuangjia Instrument Co., Ltd., China) with different parameter: temperature $\left(50-90{ }^{\circ} \mathrm{C}\right)$, ultrasonic power $(50-200 \mathrm{~W})$ and time (5-25 $\mathrm{min})$. Then, the extract was centrifuged at $3000 \mathrm{rpm}$ for $20 \mathrm{~min}$ and the yield of inulin was determined. The total sugar was measured by the phenol-sulfuric acid method, and the reducing sugar content using the DNS method. The yield of total inulin was calculated as follows (1):

$$
y=\frac{x_{1}-x_{2}}{w} \times 100 \%
$$

Where $x_{1}$ is the content of total sugar content, $x_{2}$ is the content of reducing sugar content, and $w$ represent dried sample weight.

\subsection{Comparison of three different extraction methods}

The yield of inulin from experimental group (ultrasonic-assisted extraction) and control group (hot water and microwave extraction) were compared with same parameter: $70^{\circ} \mathrm{C}$ for $30 \mathrm{~min}$. Moreover, the ultrasonic power $140 \mathrm{~W}$ and the microwave power $320 \mathrm{~W}$.

\subsection{Single-factor experiments}

The yield of inulin was evaluated through determining the effect of temperature, ultrasonic power and time during the ultrasonic-assisted extraction procedure by a single-factor design. One factor was changed while the other factors were kept constant in each experiment.

\footnotetext{
* Corresponding author: liuhe2069@163.com
} 


\subsection{Box-Behnken Design (BBD)}

Experiments were established based on BBD with three factors at three levels. Three parameters including temperature $\left({ }^{\circ} \mathrm{C}\right)$, ultrasonic power $(\mathrm{W})$ and time (min) were chosen as variables based on the results of single-factor experiments and named as $\mathrm{X} 1, \mathrm{X} 2$, and $\mathrm{X} 3$, respectively. Extraction yield (Y) was taken as the response of the design experiments. Table 1 lists the ranges of independent variables and their levels.

Table 1. Independent variables and their levels used for BBD

\begin{tabular}{|c|c|c|c|}
\hline \multirow{2}{*}{ Independent variables } & \multicolumn{3}{|c|}{ Levels } \\
\cline { 2 - 4 } & -1 & 0 & 1 \\
\hline ultrasonic temperature (X1) & 70 & 80 & 90 \\
\hline ultrasonic power (X2) & 80 & 110 & 140 \\
\hline ultrasonic time (X3) & 10 & 15 & 20 \\
\hline
\end{tabular}

\subsection{Statistical analysis}

The data were presented as the mean $\pm \mathrm{SD}$ and evaluated by one-way analysis of variance (ANOVA). Difference was considered to be statistically significant if $p<0.05$. All statistical analyses were carried out by IBM SPSS Statistics 20.

\section{Results and discussions}

\subsection{Effects of different extraction methods on inulin yield}

It can be seen from Table 2 that the yield of ultrasonic-assisted extraction was $62.07 \%$, followed by $40.85 \%$ microwave and $27.42 \%$ hot water extraction. Compared with the hot water extraction, ultrasonic-assisted extraction significantly increased $34.65 \%$, and it was non-destructive for inulin and benefited from a shorter extraction time [6].

Ultrasonic wave created and collapsed more bubble when it passed through the liquid medium, thus leading to microjet formation and acoustic streaming. Due to the swelling of the materials and enlargement of the pores, it enhanced the extraction yield of inulin [7].

Table 2. Comparison of three extraction methods

\begin{tabular}{|c|c|}
\hline Extraction methods & Inulin yield (\%) \\
\hline Hot water & $27.42 \pm 0.42$ \\
\hline Microwave & $40.85 \pm 0.28$ \\
\hline Ultrasonic-assisted & $62.07 \pm 0.39$ \\
\hline
\end{tabular}

\subsection{Effect of three parameters on the yield of inulin}

The effect of temperature on the yield of inulin was shown in Fig.1(A). Ultrasonic parameters were as follows: ultrasonic power $110 \mathrm{~W}$ and $15 \mathrm{~min}$. The yield of inulin increased with the increasing extraction temperature and reached the critical value $(74.7 \pm 1.6 \%)$ when extraction temperature was $80^{\circ} \mathrm{C}$, and then the curve began to decrease due to the destroyed of the inulin structure.

The effect of different ultrasonic power on the yield of inulin was shown in Fig.1(B). The results indicated that the maximum yield of inulin $(69.8 \pm 2.2 \%)$ was reached when ultrasonic power was $110 \mathrm{~W}$, and then it began to decrease, as generated a large number of bubbles and weakened the scattering chemical likely caused the local solution to warm up thereby partly degradation of the inulin structural [8].

A

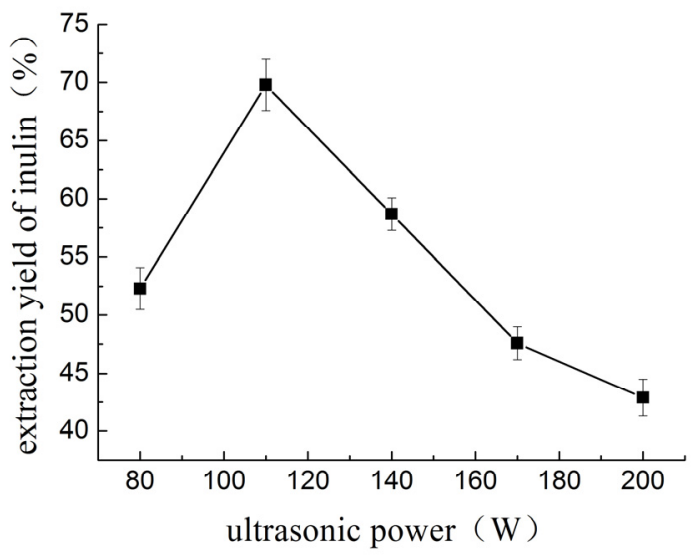

B

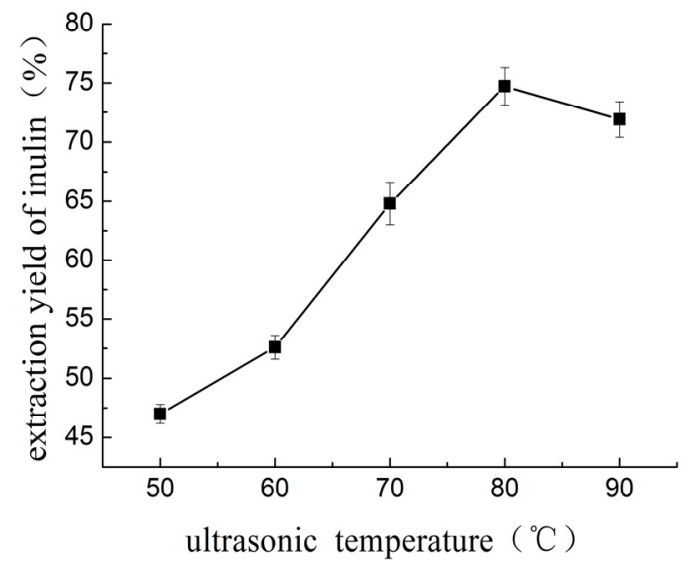

C

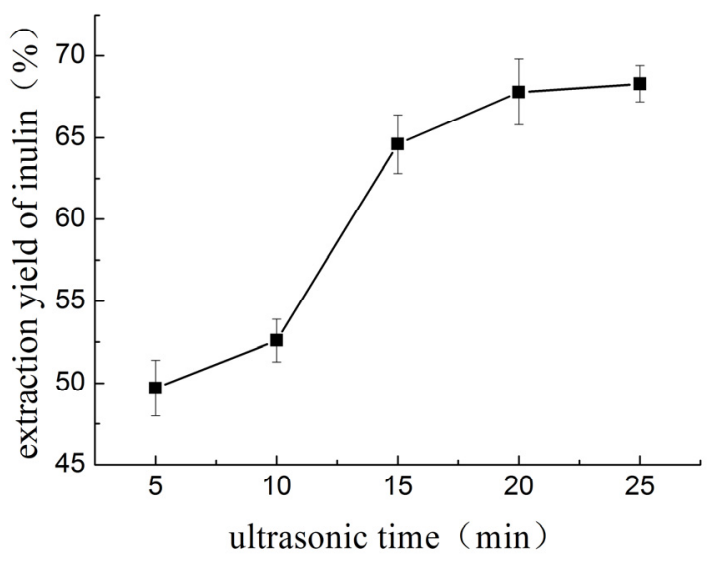

Fig. 1. Effect of temperature (A), ultrasonic power (B) and time $(\mathrm{C})$ on the yield of inulin

The effect of time on extraction yield of inulin at $70{ }^{\circ} \mathrm{C}$ 
and $110 \mathrm{~W}$ was shown in Fig.1(C). The yield increased with the increasing time and reached the value $(67.8 \pm$ $2 \%$ ) at $20 \mathrm{~min}$, and then the upward trend was obviously reduced and tended to be steady. Time was a critical factor that would affect the extraction efficiency and selectivity of the fluid. It was reported that a long extraction time presents a positive effect on the production of polysaccharides, but excessive extraction could waste time and energy [9].

Table 3. BBD and response values of the yield of inulin

\begin{tabular}{|c|c|c|c|c|}
\hline Number & $\begin{array}{c}\mathrm{X}_{1} \\
\left({ }^{\circ} \mathrm{C}\right)\end{array}$ & $\begin{array}{c}\mathrm{X}_{2} \\
(\mathrm{~W})\end{array}$ & $\begin{array}{c}\mathrm{X}_{3} \\
(\mathrm{~min})\end{array}$ & $\begin{array}{c}\text { Yield } \\
(\%)\end{array}$ \\
\hline 1 & $0(80)$ & $0(110)$ & $0(15)$ & 82.21 \\
\hline 2 & $-1(70)$ & $0(110)$ & $1(20)$ & 63.51 \\
\hline 3 & $1(90)$ & $1(140)$ & $0(15)$ & 65.93 \\
\hline 4 & $0(80)$ & $0(110)$ & $0(15)$ & 82.76 \\
\hline 5 & $0(80)$ & $-1(80)$ & $1(20)$ & 77.37 \\
\hline 6 & $-1(70)$ & $1(140)$ & $0(15)$ & 49.72 \\
\hline 7 & $-1(70)$ & $-1(80)$ & $0(15)$ & 53.25 \\
\hline 8 & $1(90)$ & $0(110)$ & $1(20)$ & 67.11 \\
\hline 9 & $0(80)$ & $0(110)$ & $0(15)$ & 84.25 \\
\hline 10 & $1(90)$ & $0(110)$ & $-1(10)$ & 74.83 \\
\hline 11 & $0(80)$ & $1(140)$ & $1(20)$ & 83.18 \\
\hline 12 & $0(80)$ & $-1(80)$ & $-1(10)$ & 73.36 \\
\hline 13 & $-1(70)$ & $0(110)$ & $-1(10)$ & 55.45 \\
\hline 14 & $0(80)$ & $0(110)$ & $0(15)$ & 81.55 \\
\hline 15 & $0(80)$ & $0(110)$ & $0(15)$ & 83.69 \\
\hline 16 & $0(80)$ & $1(140)$ & $-1(10)$ & 67.24 \\
\hline 17 & $1(90)$ & $-1(80)$ & $0(15)$ & 59.31 \\
\hline
\end{tabular}

\subsection{Statistical analysis and the model fitting}

There were a total of 17 runs for optimizing each parameter in the BBD. The corresponding results and the design matrix of RSM experiments to determine the effects of the three independent variables were shown in Table 3. The mathematical model describing the extraction yield of inulin (Y) as a function of the test independent variables over their selected ranges was given by Eq. (2):

$$
\begin{aligned}
& y=82.89+5.66 X 1+0.35 X 2+2.54 X 3 \\
& +2.54 X 1 X 2-3.95 X 1 X 3+2.98 X 2 X 3 \\
& -17.95 X 1^{2}-7.89 X 2^{2}+0.28 X 3^{2}
\end{aligned}
$$

tested for their significance using variance (ANOVA), and the analysis results of fit statistics of yield (Y) for predictive model were shown in Table 3. In our research, the F-value $(\mathrm{F}=30.4)$ and $\mathrm{P}$-value $(\mathrm{P}<0.001)$ implied that the model was extremely significant and the lack-of-fit F-value of 16.53 was also significant which meant that the model was sufficiently accurate for predicting the relevant response. The quadratic regression model showed that $\mathrm{R}^{2}$ and adj- $\mathrm{R}^{2}$ were 0.9751 and 0.9430 which indicated that $97.51 \%$ of the variation could be represented by the fitted model and $94.30 \%$ of the variation were explained by the model. It could be seen from Table 4 that X1, X3, X13, X12, X22 were important factors in the inulin yield with very small $\mathrm{P}$ values $(\mathrm{P}<0.05)$.

\subsection{Interpretation and optimization of response surface model}

The three-dimensional response surface could show a visual interaction between two tested variables and the relationships between each variable levels and related responses in the experiment, which was also used to determine the optimum conditions (Fig.2).

\subsection{Verification of predictive model}

The suitability of the model equation for predicting the optimum response values was tested using the optimal conditions. Because the optimal values were difficult to operate in the actual experiments, they were carried out with slight modifications: $82^{\circ} \mathrm{C}, 120 \mathrm{~W}$ and $18 \mathrm{~min}$. Under these conditions, the experiment yield of inulin was $82.93 \pm 1.03 \%$, which was not significantly different ( $\mathrm{P}>0.05)$ from the predicted value of $84.29 \%$. This result proved that the model designed in this study was valid.

\section{Conclusions}

Ultrasound-assisted extraction, which was an efficient and time-saving extraction technique, could be used to improve the extraction yield of inulin. The ultrasonic-assisted extraction (62.07 $\pm 0.39 \%)$ was superior to the microwave $(40.85 \pm 0.28 \%)$ and hot water extraction $(27.42 \pm 0.42 \%)$. By optimized variables by $\mathrm{RSM}$, the optimal yield of inulin was $82.93 \pm 1.03 \%$ at $82^{\circ} \mathrm{C}, 120 \mathrm{~W}$ and $18 \mathrm{~min}$, which was coincided with the predicted value of $84.29 \%$ closely.

The regression coefficients of Eq. (2) calculated and

Table 4. Results of ANOVA of regression model for the yield of inulin $(* \mathrm{p}<0.05$ significant; $* * \mathrm{p}<0.01$ extremely significant).

\begin{tabular}{|c|c|c|c|c|c|}
\hline Source & Sum of Squares & $\mathrm{df}$ & Mean Square & F Valve & P-valve Prob $>\mathrm{F}$ \\
\hline Model & 2123.82 & 9 & 235.98 & 30.4 & $<0.001^{* *}$ \\
\hline A & 255.95 & 1 & 255.95 & 32.97 & $<0.001^{* *}$ \\
\hline B & 0.97 & 1 & 0.97 & 0.12 & 0.735 \\
\hline C & 51.46 & 1 & 51.46 & 6.63 & $0.037^{*}$ \\
\hline AB & 25.76 & 1 & 25.76 & 3.32 & 0.111 \\
\hline AC & 62.25 & 1 & 62.25 & 8.02 & $0.025^{*}$ \\
\hline BC & 2.79 & 1 & 2.79 & 4.58 & 0.070 \\
\hline
\end{tabular}




\begin{tabular}{|c|c|c|c|c|c|}
\hline $\mathrm{A} 2$ & 1356.79 & 1 & 1356.79 & 174.78 & $<0.001^{* *}$ \\
\hline $\mathrm{B} 2$ & 262.01 & 1 & 262.01 & 33.75 & $<0.001^{* *}$ \\
\hline $\mathrm{C} 2$ & 0.34 & 1 & 0.34 & 0.044 & 0.840 \\
\hline Residual & 54.34 & 7 & 7.76 & & $0.014^{*}$ \\
\hline Lack of fit & 49.58 & 3 & 16.53 & 13.87 & \\
\hline Pure error & 4.76 & 4 & 1.1 & & \\
\hline Cor total & 2178.16 & 16 & & & \\
\hline C.V.\% & 3.93 & & & & \\
\hline $\mathrm{R}^{2}$ & 0.9751 & & & & \\
\hline Adj-R & 0.9430 & & & & \\
\hline
\end{tabular}
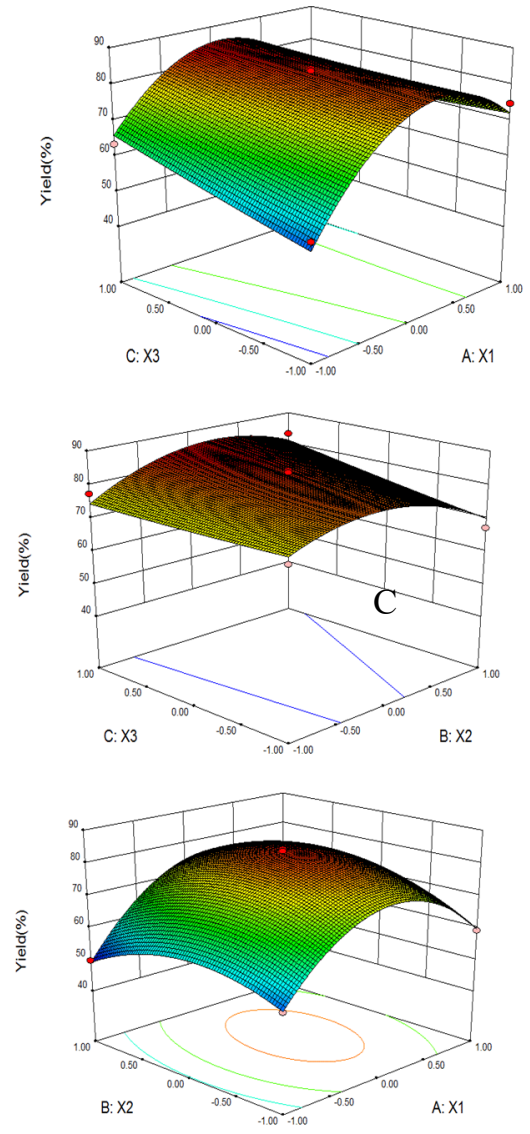

Fig. 2. The 3-D plots of variables (X1: temperature; X2: ultrasonic power; X3: time) on the yield of inulin.

This work was financed by the National Natural Science Foundation of China (31471621 and 31701618). We thank prof. Liu He (Bohai University, CN) for his critical review of the manuscript.

\section{References}

1. G. Schaafsma, JL. Slavin, Compr. Rev. Food 14, 37(2015)

2. W. Li, J. Zhang, C. Yu, Q. Li, F. Dong, G. Wang, G. Gu, Z. Guo, Carbohydr. Polym 121, 315(2015)

3. M. Roberfroid, J. Slavin, Critl. Rev. Food. Sci 40, 461(2000)

4. CM. Galanakis, Food Bioprods Process 91, 575(2013)
5. Y. Tao, D. Wu, QA. Zhang, DW. Sun, Ultrason. Sonochem 21, 706(2014)

6. Y. Xu, L. Zhang, Y. Yang, X. Song, Z. Yu., Carbohydr. Polym 117, 895(2015)

7. C. Quan, Y. Sun, J. Qu, Can. J. Chem. Eng 87, 562(2009)

8. AV. Filgueiras, JL. Capelo, I. Lavilla, C. Bendicho, Talanta 53, 433(2000)

9. XJ. Hou, W. Chen, Carbohydr. Polym 72, 67(2008) 